\title{
Identification of a locus involved in the utilization of iron by Actinobacillus pleuropneumoniae
}

\author{
Ni Chin ${ }^{a}$, Joachim Frey ${ }^{b}$, Chao-Fu Chang ${ }^{a}$, Yung-Fu Chang ${ }^{a, *}$ \\ ${ }^{a}$ Diagnostic Laboratory, College of Veterinary Medicine, Cornell University, Ithaca, NY 14853, USA \\ ${ }^{b}$ Institute for Veterinary Bacteriology. University of Berne, Länggass-Strasse 122, CH-3012 Berne. Switzerland
}

Received 8 February 1996; revised 4 June 1996; accepted 7 June 1996

\begin{abstract}
The cloned afu locus of Actinobacillus pleuropneumoniae restored the ability of an Escherichia coli K-12 mutant (aroB) to grow on iron-limited media. DNA sequence analysis of the fragment showed that there are three genes designated afuA, afuB and $a f u C$ (Actinobacillus ferric uptake) that encode products similar to the SfuABC proteins of Serratia marcescens, the HitABC proteins of Haemophilus influenzae, the FbpABC proteins of Neisseria gonorrhoeae and the YfuABC proteins of Yersinia enterocolitica. The three genes encode a periplasmic iron-binding protein (AfuA), a highly hydrophobic integral cytoplasmic membrane protein with two consensus permease motifs (AfuB) and one hydrophilic peripheral cytoplasmic membrane protein with Walker ATP-binding motifs (AfuC), respectively. This system has been shown to constitute a periplasmic binding protein-dependent iron transport system in these organisms. The afuABC operon is locating approximately $200 \mathrm{bp}$ upstream of apxIC gene, but transcribed in opposite direction to the ApxI-toxin genes.
\end{abstract}

Keywords: Actinobacillus pleuropneumoniae; Iron-uptake operon; afuABC; ApxI

\section{Introduction}

The low concentration of free iron, an essential nutrient for bacteria, on mucous membranes and in tissues is one of the first lines of host defense against bacterial infection. The presence of iron-binding proteins in the body fluids, such as transferrin, lactoferrin, haem, haemoglobin, and ferritin further serves to maintain low free- $\mathrm{Fe}$ concentrations, inhibiting bacteria growth [1]. To sequester the limited iron from the host, bacteria have evolved several mechanisms, such as the secretion of siderophores and iron chelators which compete with lactoferrin and transferrin

\footnotetext{
* Corresponding author.
}

for iron. Iron-repressible outer membrane proteins (IROMP) that scrve as receptors for iron-siderophore complexes are essential for iron uptake have been identified in many pathogenic bacteria [2], including Actinobacillus pleuropneumoniae [3].

A. pleuropneumoniae obtains iron from haem compounds [3] via the production hemolysins [4,5], and membrane-bound transferrin-specific receptors [6]. A. pleuropneumoniae probably binds the iron-loaded transferrin molecule to its surface and then, transports the iron from the transferrin into the cells. However, a mechanism for the transfer of iron from the transferrin to the bacterium has not been elucidated.

In this study, we reported the cloning and se- 


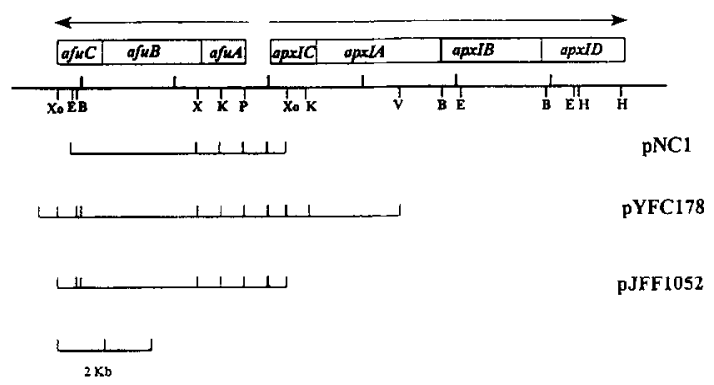

Fig. 1. Partial restriction enzyme map of afu and apxI operons of A. pleuropneumoniae. The inserted DNA from $\mathrm{pNC} 1$ and pJFF1052 were completely sequenced. E, EcoRI; V, EcoRV; B, $B g I I \mathrm{I} ; \mathrm{K}, K p n \mathrm{I} ; \mathrm{H}$, HindIII; P, PstI; X, XbaI; Xo, Xhol. Arrows indicate direction of expression.

quence analysis of an iron utilization system in $A$. pleuronpneumoniae that is very similar to the periplasmic-binding protein-dependent transport system in Serratia marcescens [7,8], Haemophilus influenzae $[9,10]$, Neisseria gonorrhoeae $[11,12]$ and Yersia enterocolitica (unpublished data). This locus, termed afu (Actinobacillus ferric uptake) is located upstream of $A p x I C A B D$ gene cluster, but is transcribed in opposite direction to the hemolysin gene (apxIC). Complementation analysis showed that this locus could restore the ability of an $E$. coli aroB mutant to grow on iron-depleted medium.

\section{Materials and methods}

\subsection{Bacterial strains, plasmids, growth conditions and DNA preparation}

A. pleuropneumoniae serotype 1 to 12 reference strains described previously [13] were grown in brain-heart infusion broth (BHI, Difco Laboratories) supplemented with $0.1 \%$ NAD or on chocolate agar. The $E$. coli strains, TB1, ara $\triangle($ lacproAB)rpsL $\Phi 80 \mathrm{~d} l a c \mathrm{Z} \triangle \mathrm{M} 15 \quad h s d R 17 \quad\left(\mathrm{r}^{-} \mathrm{m}^{+}\right)$, $\mathrm{H} 1443$, aro $\mathrm{D}$ ara $\mathrm{D} 139 \Delta$ lac $\mathrm{U} 169$ rpsL150 rela1 deoC1 ptsF25 rbsR thi flb5301, and DH5 $\alpha$ \$80dlac$\mathrm{Z} \triangle \mathrm{M} 15 \triangle($ lacZYA-argF)U169 endA1 recAlhsd $17\left(\mathrm{r}_{\mathrm{k}}{ }^{-} \mathrm{m}_{\mathrm{k}}{ }^{+}\right)$deo $\mathrm{R}$ thi-1 sup E44 $\gamma^{-}$gyrA96 relAl were grown in Luri-Bertani (LB) broth or LB agar. Antibiotics were used as appropriate for selection or maintenance of plasmids, ampicillin 40 $\mu \mathrm{g} / \mathrm{ml}$, and kanamycin $50 \mu \mathrm{g} / \mathrm{ml}$. X-gal (5-bromo-in- dolyl- $\beta$-D-galactopyranoside) was added to agar plates to $40 \mu \mathrm{g} / \mathrm{ml}$. Plasmids pYFC126 and pYFC127 contains apxICA genes and a segment upstream apxIC from serotype 1 and 5, respectively [14]. $\mathrm{pNCl}$ contains $a f u A B$ and partial af $u C$ genes. Plasmid pYFC178 contains afuABC genes subcloned from the phage clone, $\lambda y f c 40$ originating from $A$. pleuropneumoniae serotype 5 (Fig. 1). Plasmid pJFF 1052 contains afuABC in $\mathrm{pBS}$ (same direction to $\mathrm{P}_{\text {lac }}$ ) (Fig. 1) and plasmid pSZ1 contains $s f u A B C$ in $\mathrm{pRR} 322$ [7].

\subsection{DNA isolation and construction of a genomic and} sub-genomic library of $A$. pleuropneumoniae $D N A$ in $\lambda$-dash and screening

A. pleuropneumoniae genomic DNA from different serotypes was prepared as previously described [13]. A lamda-dash library was constructed by using the genomic DNA from a serotype 5 strain as previously described [5]. A subgenomic library from serotype 5 was also constructed using $B g / \mathrm{II}$ and PstI digested DNA fragments separated by agarose gel electrophoresis. The 4.5-6 kbp fragments were ligated into pHG165 digested with BamHI and Sall. The bacteriophage and subgenomic libraries were screened by hybridization using a probe (a $1033 \mathrm{bp}$ PstI-Xhol DNA fragment from pYFC126) contaning the partial apxIC gene and its upstream region from $A$. pleuropneumoniae serotype 5 [14].

Table 1

The conserved EAA motifs from the periplasmic permeases of $A$. pleuropneumoniae, $S$. marcensens, $H$. influenzae, $N$. gonorrhoeae and $Y$. enterocolitica

\begin{tabular}{lll}
\hline Protein $^{\mathrm{a}}$ & Residue & Conserved sequence \\
\hline AfuB (N) & 303 & EEASYTLRANRYQTFYNI IFP \\
SfuB (N) & 167 & EDVATSLGSRPPAVFFRVVLP \\
HitB (N) & 148 & EEVS I SLGKSPVYTFWYAIFP \\
YfuB (N) & 168 & EDAAASLGSTPSAVFF HVVLP \\
FbpB (N) & 153 & EEVSLSLGKSRLQTFF SAILP \\
AfuB (C) & 581 & EASLSLKGSSLKTIWF IVFPL \\
SfuB (C) & 428 & ENVARSLGKSPAQA iWSTTIR \\
HitB (C) & 410 & EKVGGSLGRNPFYIFRTITLP \\
YfuB (C) & 429 & ENVARSLGKTPTQAIWSTTMR \\
FbpB (C) & 426 & EQVGATLGRGHF F IFRTLVLP \\
\hline
\end{tabular}

"The sequence were taken from the following sources: $a f u B$ (this study), $s f i B$ [7], $f b p b$ [12], hitb [9] and $y f u B$ (unpublished data). 

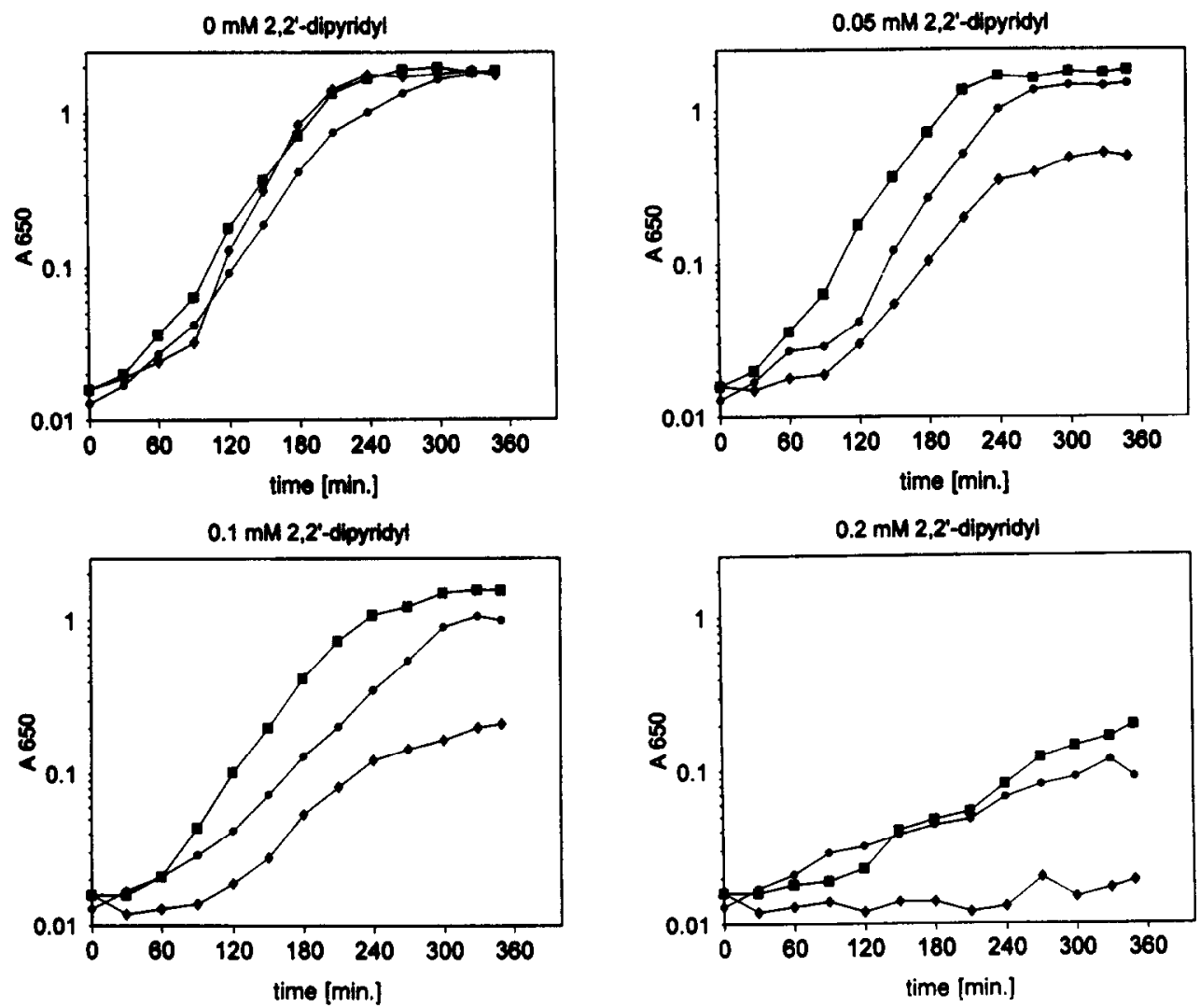

Fig. 2. Growth rates of the aroB mutant H1443 and the complemented mutant in LB containing different concentrations of 2,2'-dipyridyl. Growth was monitored with a spectrophotometer at A650. The cultures were inoculated to $A_{650}=0.01$ with fresh precultures. $\downarrow$, H1443 (aroB); •, H1443 [pJFF1052 $\left.\left(a f u A^{+} B^{+} C^{+}\right)\right] ; \mathbf{\square}, \mathrm{H} 1443$ [pSZ1 $\left.\left(s f u A^{+} B^{+} C^{+}\right)\right]$.

\subsection{DNA sequencing and analysis}

Plasmid DNA for cycle DNA sequencing was isolated with a mini-kit from Qiagen (Chatsworth, CA). The nucleotide sequence was determined by an automated fluorescence procedure based on the Sanger dideoxy chain termination method using a Taq DyeDeoxy Terminator Cycle Sequencing kit (Applied Biosystem, Inc.). DNA sequences were determined by using double-stranded DNA templates (pNCl, pYFC178 and pJFF1052). Oligonucleotide primers based on the DNA sequence in this study were prepared by the Analytical and Synthetic Facility, Cornell University. Both strands of the cloned DNA were completely sequenced.

\subsection{Trans-complementation of E. coli aroB}

E. coli $\mathrm{H} 1443$ (aroB mutant) transformed with plasmid pJFF1052 or with plasmid pSZ1 and $E$. coli $\mathrm{C} 600\left(\mathrm{aroB}^{+}\right)$was inoculated in LB broth or in LB broth supplemented with $0.05,0.1,0.2$, or 0.4 mM 2,2'-dipyridyl (NBD) (Sigma, St Louis, MO) and the growth was monitored by measuring the absortion $\mathrm{A}_{650}$ in a spectrophotometer.

\subsection{Southern blotting, hybridization}

A $\boldsymbol{P}_{\text {st I I }}$ and $X b a \mathrm{I}$ DNA fragment from $\mathrm{pNCl}$ containing the $a f u A$ gene was isolated from agarose gel and labeled with $\left[{ }^{32}\right.$ P $]$ dATP by nick-translation. Filters were hybridized in $45 \%$ formamide, $5 \times$ SSC, $5 \times$ Denhardt's solution, and $100 \mu \mathrm{g} / \mathrm{ml}$ sheared calf 


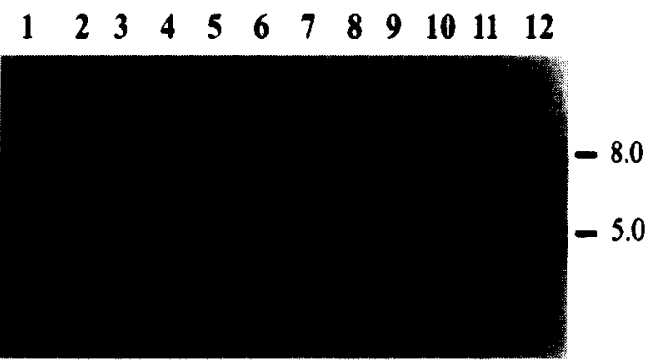

Fig. 3. Southern blotting analysis of 12 serotypes reference strains of $A$. pleuropneumoniae. The lanes are labeled according to the serotype number. The filter was hybrized and washed as described in Section 2. Molecular mass in kbp as indicated at the right.

thymus DNA for $12 \mathrm{~h}$ at $37^{\circ} \mathrm{C}$. Filters were then washed twice with $2 \times \mathrm{SSC}-0.1 \%$ SDS and twice with $0.2 \%$ SSC $-0.1 \%$ SDS at room temperature $[4,13]$. The final wash was with $0.16 \%$ SSC $-0.1 \%$ SDS at $37^{\circ} \mathrm{C}$.

\section{Results and discussion}

\subsection{Nucleotide sequences of the afuA, afuB and afuC genes}

Plasmid $\mathrm{pNCl}$ and phage clone $\lambda \mathrm{yfc} 40$ were se- lected from subgenomic and $\lambda$-dash library, respectively. Plasmid pNC1 contains a $5.0 \mathrm{~kb}$ of the $A$. pleuropneumoniae serotype 5 chromosome, cloned into the Sall and BamHI sites of pHG165 $[4,14]$. $\lambda y f c 40$ was digested with $S a l l$ and a $9 \mathrm{~kb}$ fragment was ligated into pBluescriptII- $\mathrm{SK}^{-}$, to obtain pYFC178. Plasmid pJFF1052 contains a $5.8 \mathrm{~kb}$ chromosomal fragment of $A$. pleuroneumoniae serotype 1 (strain 4074) including the 5 '-terminal part of the apxIC and a $5.4 \mathrm{~kb}$ segment upstream apxIC cloned into the XhoI site of pBluescriptII-SK ${ }^{-}$ (Fig. 1). The sequences of both clones showed three open reading frames with high similarities to the sf $u A B C$ gene cluster of $S$. marcescens [7]. In analogy to $s f u A B C$, the three reading frames were designated $a f u A$, $a f u B$, and $a f u C$, respectively (Fig. 1). The DNA sequence of afuABC genes from $A$. pleuropneumoniae serotype 1 and 5 are identical.

The deduced amino acid sequence of AfuA deduced from the nucleotide sequence of afuA contains a typical signal sequence found in exported proteins. Cleavage of the signal peptide most likely occurs between the $A$ and $K$ residue at positions 27 and 28. AfuB is proposed to function as a cytoplasmic membrane permease and is composed of 663 amino acids, most of which are hydrophobic. Two sequences that match the consensus permease EAA motifs (EAA- - -G- - . - . . - -I-LP) are found (Table

Table 2

Comparison of non-polar membrane transport proteins containing sequences homologous to nucleotide-binding domains

\begin{tabular}{|c|c|c|}
\hline Protein & Residue & Conserved sequence \\
\hline AfuC (Ap) & 136 & I S GGQQQRVALARALVLK-PK-----VLILDE PLSNLDAN LRRSMREKIRE \\
\hline $\mathrm{SfuC}(\mathrm{Sm})$ & 136 & 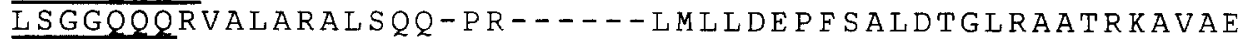 \\
\hline $\operatorname{HiTC}(\mathrm{Hi})$ & 146 & LSGGQQQRVALARA LA P N $-P E------L I L L D E P F S A L D E H L R Q Q$ I RQEMLQ \\
\hline YfuC (Ye) & 136 & ISGGQQQRVALARALGQR-PA----- LMLLVEPFSTLDTALRASTRKAVAE \\
\hline $\mathrm{fBPc}(\mathrm{Ng})$ & 138 & LSGGQQQRVALARALAPD-PE----- - L I LLDEPF SALDEQLRRQIREDM I A \\
\hline FecE & 139 & LSGGQRQRAFLAMVLAQNTP---- - - VVLLDEPTTYLDIN HQVDLMRLMGE \\
\hline FhuC & 141 & LSGGERQRAWI AMIVAQDDS-R--- - - C L LLDEPTSALD I A HQVDVLSLVHR \\
\hline BtuD & 127 & LSGGEWQRVRLAAVVLÄITPQANPAGQLLLLDEPMNSLDVAQQSALDKILSA \\
\hline HisP & 154 & LSGGQQQRVS I ARAL-AMEPD---- - - VLLF DEPTSALDPE L V GEVLRIMQQ \\
\hline MalK & 134 & LSGGQRQRVA I GR JLVA-EPS--- - - VFLLDEPLSNLDAALRVQMR I E I SR \\
\hline PstB & 152 & LSGGQQQRLCIARGL-A I RPE- - - - VLLLDEPC SALDP I STGR IEELITE \\
\hline OppD1 & 165 & FSGGQCQR I G I ARAL - I LEPK------ I I I CDE PVSALDVS I QAQVVNLLQQ \\
\hline OppD2 & 169 & FSGGMRQRVMIAMALL-CRPK----- - LLIADEPTTALDVTVQAQIMTLLNE \\
\hline OppD (St) & 167 & FSGGMRQRVMIAMALL-CRPK------ILIADEPTTALDVTVQAQIMTLLNE \\
\hline $\operatorname{RbsA}(\mathrm{N})$ & 144 & LSIGDQQMVEIAKVLSF-ESK -....- -VIIMDEPTSALTDTETESLFRVIRE \\
\hline
\end{tabular}

${ }^{*}$ Proteins were from $E$ c coli unless otherwise indicated. A glycine-glutamine-rich sequence, LSGGQQQ (Linker peptide) is underlined. Ap, $A$. pleuropneumoniae (this study); $\mathrm{Hi}, H$. influenzae [9]; $\mathrm{Ng}, N$. gonorrhoeae [12]; Sm, S. marcescens [7]; St, S. tryphimurium; Ye, Y. enterocolitica (unpublished data). $\mathrm{N}$, amino-terminal half of the polypeptide. 
1). These two motifs are suggested to be located on cytoplasmic loops that interact with the ATP-binding protein $[16,17]$. AfuC shows strong similarities to the nucleotide-binding proteins of ABC (ATP Binding Cassette) transporters [18] (Table 2). A comparison of AfuA, AfuB and AfuC with homologous proteins are presented in Table 3. A. pleuropneumoniae together with other pathogenic bacteria possess a siderophore-independent mechanisms for iron sequestration $([7,10,12]$, this study). In $N$. gonorrhoeae and $N$. meningitidis, two proteins (Tbp1 and Tbp2) are responsible for binding transferrin to the cell surface [15]. Similarly, the genes for two transferrin binding proteins ( $\mathrm{Tbp} 1$ and $\mathrm{Tbp} 2$ ) have been cloned and sequenced in A. pleuropneumoniae [6]. In Neisseria species, the iron can be removed from transferrin or lactoferrin to the periplasmic space, and carried by $\mathrm{Fbp}$ to transport the iron molecule into the cells [15]. It has also been suggested that the iron is diffusible through the $E$. coli porin to the periplasm that is independent of the transferrin receptor [10]. The mechansim of iron transport from porcine transferrin into A. pleuropneumoniae is unknown. However, the presence of afu operon homologs in $H$. influenzae [9,10], $S$. marcescens [7], and $N$. gonorrhoeae $[11,12]$ suggests that the function of this operon may be involved in high-affinity iron acquisition from the host environment.

\subsection{Complementation of the iron transport negative E. coli strain}

To examine the function of the $A$. pleuropneumoniae transport genes in iron uptake, iron transport

Table 3

Comparision of AfuA, AfuB and AfuC with homologous proteins

\begin{tabular}{lllll}
\hline Organism & Gene & $\mathrm{A}(\mathrm{S} / \mathrm{I})^{\mathrm{a}}$ & $\mathrm{B}(\mathrm{S} / \mathrm{I})^{\mathrm{a}}$ & $\mathrm{C}(\mathrm{S} / \mathrm{I})^{\mathrm{a}}$ \\
\hline S. marcescens $^{\mathrm{b}}$ & sfu & $47.6 / 23.1$ & $50.5 / 20.0$ & $50.8 / 33.0$ \\
H. influenzae $^{\mathrm{b}}$ & hit & $45.5 / 25.0$ & $52.1 / 23.9$ & $56.2 / 33.5$ \\
Y. enterocolitica $_{\text { }}$ & yfu & $45.7 / 21.5$ & $49.7 / 20.0$ & $55.4 / 31.9$ \\
N. gonorrhoeae $^{\mathrm{b}}$ & fbp & $45.6 / 22.8$ & $51.4 / 22.9$ & $55.6 / 32.2$ \\
\hline
\end{tabular}

${ }^{a}$ Percent similar/identical residues ( $S$, similarity; $\mathbf{l}$, identity). Percent similar residues assuming that the following amino acid pairs are equivalent; $\mathrm{I}$ and $\mathrm{V}, \mathrm{S}$ and $\mathrm{T}, \mathrm{E}$ and $\mathrm{D}, \mathrm{K}$ and $\mathrm{R}, \mathrm{F}$ and $\mathrm{Y}$. ${ }^{\mathrm{b}}$ The sequence were taken from the following sources: afuABC (this study), sfuABC (7), $f b p(11,12)$, hit $(9,10)$ and $y f u A B C$ (unpublished data). was studied in $E$. coli aroB mutant strain $\mathrm{H} 1443$ carrying cloned af $A B C$ genes. As shown in Fig. 2, the af $A B C$ genes confer the $E$. coli aro $B$ mutant which is unable to synthesise enterochelin, to grow in iron-limiting medium. In medium supplemented with $0.05 \mathrm{mM}$ or $0.1 \mathrm{mM} 2,2^{\prime}$-dipyridyl, the strains containing the cloned af $A A B C$ genes or the $s f u A B C$ genes grow significantly faster and to a higher density than the non-complemented aro $B$ mutant (Fig.

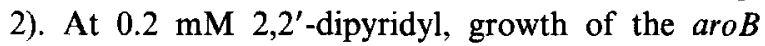
mutant was inhibited, but the complemented mutant was able to grow, albeit at a reduced growth rate. The growth rate of $E$. coli $\mathrm{C} 600\left(\right.$ aro $\left.^{+}\right)$was unaffected at these concentrations, but was generally higher in this medium compared to H1443. Addition

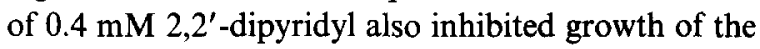
complemented transformants of $\mathrm{H} 1443$, and also reduced the growth rate of the control strain $E$. coli C600 $\left(\right.$ aro $\left.^{+}\right)$. Supplementation of medium containing $0.2 \mathrm{mM} 2.2^{\prime}$-dipyridyl with $2 \mathrm{mM} \mathrm{Fe}(\mathrm{SO})_{4}$ restored the growth rates of $\mathrm{H} 1443$ and the complemented H1443 strains. However, only partial restoration of the growth rates was observed in the

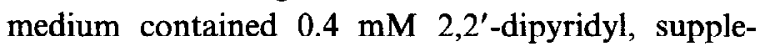
mented with $\mathrm{Fe}(\mathrm{SO})_{4}$. These results indicated that the importance of the functional af $u \mathrm{ABC}$ operon for iron acquisition by complementation of the siderophore-deficient $E$. coli $\mathrm{H} 1443$ to growth on dipyridyl-containing medium.

\subsection{Identification of the afuA gene by Southern blotting analysis}

A PstI and $X b a I$ DNA fragment containing afuA was purified, labelled with $\left[{ }^{32} \mathrm{P}\right] \mathrm{dATP}$, and used as a hybridization probe on genomic DNA of $A$. pleuropneumoniae serotypes. The results showed that the afuA DNA hybridized to one unique fragment in the DNA of the A. pleuropneumoniae serotypes 10 and $11(8 \mathrm{kbp}), 1,5$ and $9(7.8 \mathrm{kbp}), 3(7.4 \mathrm{kbp})$ and $2,4,7$, and $8(5 \mathrm{kbp})$, but not to serotype 6 (Fig. 3).

In conclusion, the afuABC operon of $A$. pleuropneumoniae is sufficient to enable an $E$. coli $\mathrm{K} 12$ (aroB) mutant to grow on iron-limited medium (4 $\mathrm{mM}$ dipyridyl). The three polypeptides deduced from the DNA sequence were similar to that of SfuABC [7], HitABC [9,10], FbpABC [11,12] and 
YfuABC. Based on these data, we hypothesized that the AfuA, AfuB and AfuC polypeptides are involved the transport of ferric iron across the cytoplasmic membrane. An efficient iron acquisition system for these pathogenic bacteria may play an important role in the pathogensis of bacterial infection.

\subsection{Nucleotide sequence accession number}

The sequence of afuABC-apxICA genes from serotype 1 and 5 has been submitted to Genbank and assigned accession numbers U05042 and U04954, respectively.

\section{Acknowledgments}

We are grateful to Helen Bell for administration assistance, to V. Braun, Tübingen, for gift of $E$. coli strain H1443 and plasmid pSZ1 and to S. Highlander, Houston, TX, for helpful discussions. This work was support by grants from the USDA Animal Health and Disease Research Program, the Pfizer Animal Health Inc. (formerly SmithKline Beecham Animal Health) to Y.F.C. and the Swiss National Science Foundation Grant 3100.39123 .93 to J.F.

\section{References}

[1] Weinberg, E.D. (1978) Iron and infection. Microbiol, Rev. 42, 45-66.

[2] Otto, B.R., Verweij-van Vught, A.M.J.J. and Maclaren, D.M. (1992) Transferrins and heme-compounds as iron sources for pathogenic bacteria. Crit. Rev. Microbiol. 18, 217-233.

[3] Deneer, H.G., and Potter, A.A. (1989) Effect of iron restriction on the outer membrane proteins of Actinobacillus (Haemophilus) pleuropneumoniae. Infect. Immun. 57, 798-804.

[4] Chang, Y.F., Young, R. and Struck, D.K. (1989) Cloning and characterization of a hemolysin gene from Actinobacillus (Haemophilus) pleuropneumoniae. DNA 8, 635-646.

[5] Frey, J., Beck, M., Stucki, U. and Nicolet, J. (1993) Analysis of hemolysin operons in Actinobacillus pleuropneumoniae. Gene $123,51-58$.
[6] Gonzalez, G.C., Yu, R.-H., Eosteck, P.R., Jr. and Schryvers, A.B. (1995) Sequence, genetic analysis, and expression of $A C$ tinobacillus pleuropneumoniae transferrin receptor genes. Mi crobiology 141, 2405-2416.

[7] Angerer, A., Gaisser, S. and Braun, V. (1990) Nucleotide sequences of the $s f u A$, sfuB, and $s f u C$ genes of Serratia marcescens suggest a periplasmic-binding protein-dependent iron transport mechanism. J. Bacteriol. 172, 572-578.

[8] Angerer, A., Klupp, B. and Braun, V. (1992) Iron transport systems of Serratia marcescens. J. Bacteriol. 174, 1378-1387.

[9] Sanders, J.D., Cope, L.D. and Hansen, E.J. (1994) Identification of a locus invlved in the utilization of iron by Haemophilus influenzae. Infect. Immun. 62, 4515-4525.

[10] Adhikari, P., Kirby, S.D., Nowalk, A.J., Veraldi, K.L., Schryvers, A.B. and Mietzner, T.A. (1995) Biochemical characterization of a Haemophilus infuenzae periplasmic iron transport operon. J. Biol. Chem. 270, 25142-25149.

[11] Berish, S.A., Mietzner, T.A., Mayer, L.W., Genco, C.A., Holloway, B.P. and Morse, S.A. (1992) Molecular cloning and characterization of the structural gene for the major iron-regulated protein expressed by Neisseria gonorrhoeae. J. Exp. Med 171, 1535-1546.

[12] Adhikari, P., Berish, S.A., Nowalk, A.J., Veraldi, K.L., Morse, S.A. and Mietzner, T.A. (1996). The $f b p A B C$ locus of Neisseria gonorrhoeae functions in the periplasm-to-cytosol transport of iron. J. Bacteriol. 178, 2145-2149.

[13] Chang, Y.F., Shi, J., Ma, D.P., Shin, S.J. and Lein, D.H. (1993) Molecular analysis of the Actinobacillus pleuropneumoniae RTX toxin-III genc cluster. DNA Cell Biol. 12, 351-362.

[14] Chang, Y.F., Wang, Y., Chin, N., Shin, S.J. and Lein, D.H. (1994) Expression and sequence analysis of an RTX-1 toxin determinant from Actinobacillus pleuropneumoniae serotype 5. Virulence mechanisms of bacterial pathogens, international symposium, June 68,1994. Abstract 38. Iowa State University, Ames, IO.

[15] Chen, C.Y., Berish, S.A., Morse, S.A. and Mietzner, T.A. (1993) The ferric iron-binding protein of pathogenic Nesseria spp. functions as a periplasmic transport protein in iron acquisition from human transferrin. Mol. Microbiol. 10, 311318.

[16] Saurin, W., Köster, W. and Dassa, E. (1994) Bacterial binding protcin-dependent permeases: characterization of distinctive signatures for functionally related integral cytoplasmic membrane proteins. Mol. Microbiol. 12, 993-1004.

[17] Kerppola, R.E. and Ames, G.F.L. (1992) Topology of the hydrophobic membrane-bound components of the histidine periplasmic permease. I. Biol. Chem. 267, 2329-2336.

[18] Higgins, C.F. (1992) ABC transporters: from microorganisms to man. Annu. Rev. Cell Biol. 8, 67-113. 